\title{
Envisioning the future of academic cartographic education
}

\author{
Georg Gartner a, Menno-Jan Kraak ${ }^{b}$ * Dirk Burghardt ${ }^{c}$, Liqiu Meng ${ }^{\mathrm{d}}$, Juliane Cron ${ }^{\mathrm{d}}$, Corné van \\ Elzakker ${ }^{\mathrm{b}}$, Britta Ricker ${ }^{\mathrm{b}}$ \\ ${ }^{b}$ University of Twente/ITC,Department of Geo-Information Processing,m.j.kraak@utwente.nl,b.a.ricker@utwente.nl, \\ c.vanelzakker@utwente.nl \\ aTU Wien, Research Group Cartography,georg.gartner@tuwien.ac.at \\ c TU Dresden, Institute of Cartography,dirk.burghardt@tu-dresden.de \\ ${ }^{d}$ Technical University of Munich, Chair of Cartography, liqiu.meng@tum.de, juliane.cron@tum.de \\ * Corresponding author
}

Keywords: Cartographic education, cartographic method, MSc cartography, cartographic competences

\begin{abstract}
Why do we teach cartography? The need for cartographic education: In our day to day life, on an individual or societal level there is a continual need or even demand for geospatial information. On an individual level this need is expressed by questions like: Where am I?, How far away is my new doctor's office?, Which route should I take to get to my destination based on current traffic patterns? Other questions may include: What is the spatial extent of my land parcel? What do I have permission to build on my parcel? On a societal level questions include: What cities suffer from high unemployment? What are the most efficient spots to build a new wind farm? Where is the optimal place to build a new road without fragmenting important species habitats? To offer answers to these questions, geographic information systems (GIS) including tools and instruments have been developed. The most important communication tool to foster decision making, as part of a GIS, is the map. Reality is too complex to comprehend with the naked eye. Therefore patterns are often missed, maps and other cartographic models are an interface between humans and the reality used to abstract, symbolized, a simplify view of the world. These maps then allow us to view spatial patterns and relationships between objects in the world. The world cannot do without maps. Why? Because they tell us about spatial issues on both local and global scale that influence our lives. How? Maps are the most effective and the most efficient tools to into and overview of geographical data which help us answer spatio-temporal questions and to provide new insight.
\end{abstract}

What is ongoing in our world? Trends in our domain: yesterday, today and tomorrow: Looking at the timeline of our domain, cartography, we could argue that after a long period where maps where seen as artifacts, maps are now considered to be interactive and dynamic (web) services, and in the near future we move to human centered cognitive map displays that are immersive and ubiquitous. Yesterday, the map could be considered an artifact, a static object, on paper or on a screen. The map stores the information and can no longer be changed. The user did not play a prominent role in map design. Today, with the internet, there has been a huge increase in data access and generation resulting in maps being produced and used especial to satisfy individual location-based queries such as 'Where am I right now' and 'How-do-I-get-there?' questions. Societal questions are answered by maps available via automated services accessible via dedicated portals. Today maps are no longer artifacts, but provided as a digital map services. However, tomorrow the map will yet again be different. We are able to sense and monitor the world real time and ubiquitously, including human users' spatial abilities, emotions, needs and requirements. With developments in interface design including more opportunities for 3d/4d/Virtual Reality/Augmented Reality Human-Computer-Interfaces are becoming even "closer" to our human processing system. Maps will increasingly become human-centered, highly interactive, dynamic and adjustable visual displays.

Purpose: What are the cartographic consequences of these developments? Required cartographic competences: The above developments have resulted in the expansion of what define the existing established cartographic method: making geospatial data and information accessible for users to foster discovery and insight into and overview of spatiotemporal data. Map design, including fundamentals such as projection, scale, generalization and symbolization, remain core to cartography. Yesterday, cartographic education was focused on how to optimally create fixed graphical representations at a defined scale constrained by the media, but with an eye for syntactical as well as graphical/aesthetical quality. Today knowledge and skills cartographers require have expanded, and they include an understanding of Spatial Data Infrastructures (SDI) that house Big Data and Data Science, Web Services, Programming, Style Definitions, Algorithms, Semantic web and Linked Data and Interactivity and other relevant technological skills. Increasingly, more attention has also been, and will have to be, paid to use and user (requirement) analysis and usability assessment. Users will simple not use cartographic services that are not enjoyable and do not help them meet their goals. We will continue to conduct usability evaluations in new sensing and map display environments. Based on technological advances and social uptake thereof, tomorrow will yet again ask for an adaption of the cartographic education and research dealing more and more with the "human" embodied experience. 
Figure 1a shows the relation among the current skills and competences a cartographer needs. In the center of the triangle the map and the cartographic method. Data, Media and Users are found around. Knowledge and skills about data handling refer to selection, integration and abstraction, as well as analysis. Media skills and knowledge are about the interface, interaction, adapted design, technology and coding. Users refers to usability (enjoyment), cognition, perception, sensors (robots) and requirements. In Figure $1 \mathrm{~b}$ the changing paradigm of the map as interface between human and reality as seen yesterday, today and tomorrow.
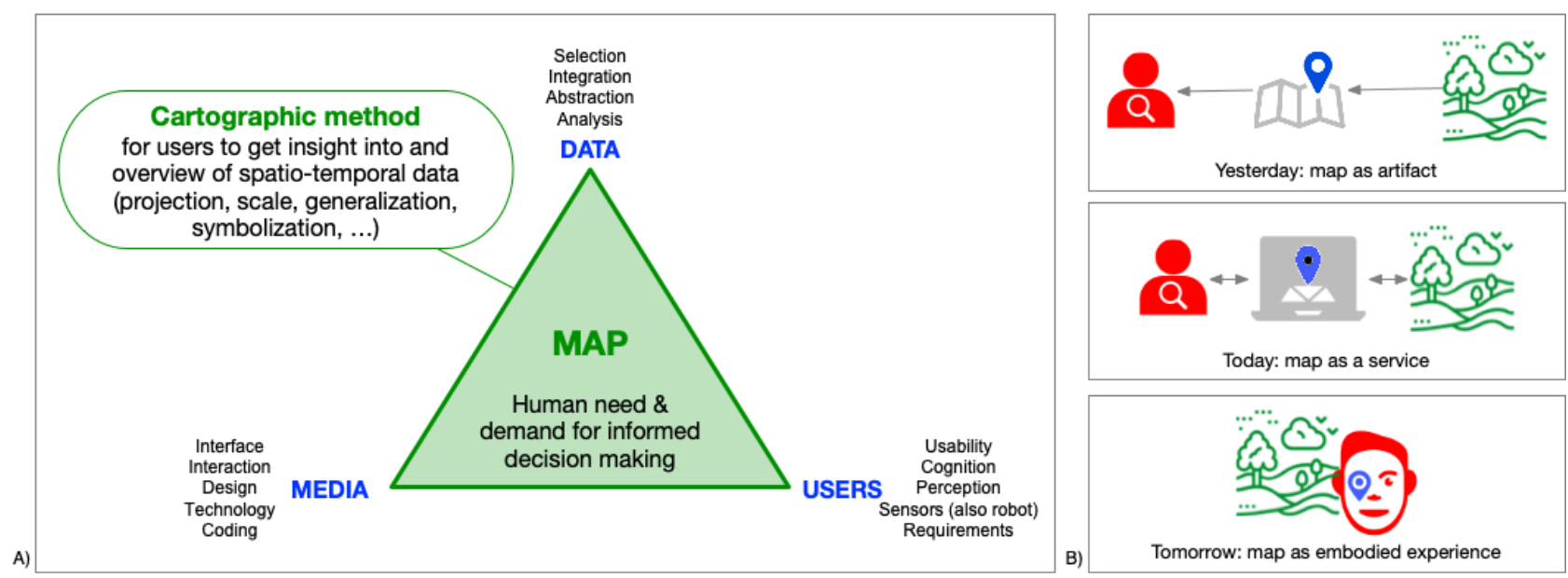

Figure 1. Required cartographic skills and competences: a) the cartographic methods; b) the changing paradigm.

How do we do it? Our MSc Cartography: The Erasmus Mundus Master of Science in Cartography program is characterized by its worldwide unique profile and comprehensive and in-depth cartographic lectures and lab works. All four partner universities (see involved authors) jointly developed and defined the learning outcomes after intensive cooperation and consultation. The program takes all theoretical as well as practical aspects of the broad and interdisciplinary field of cartography into account. Graduates of the program are able to meet the variety of requirements placed on a cartographer today.

An obvious strength of this program is the clear research-driven orientation of selected lectures, e.g. visual analytics, web and mobile cartography and the close binding of M.Sc. topics to ongoing research projects.

Students in the Cartography program learn how to develop and evaluate cartographic tools on the basis of firmly established theories and methods. The focus lays in developing and applying scientific methods and techniques to improve geo-information services for a diverse range of heterogeneous users.

Another added value of the program is its educational execution in locations across Europe, a historic center of excellence in the field of cartography, integrating it within interdisciplinary fields. Excellently educated students from this program will fill the gaps not only in the cartographic research community and geosciences, but also in other related research fields that address the global challenges as defined by bodies like the United Nations or the European Union. 